\title{
Recent Advances in Imaging Steroid Hormone Receptors in Breast Cancer
}

\author{
Manoj Kumar ${ }^{1}$, Kelley Salem ${ }^{1}$, Amye J. Tevaarwerk ${ }^{2,3}$, Roberta M. Strigel ${ }^{1,2,4}$, and Amy M. Fowler ${ }^{1,2,4}$ \\ ${ }^{1}$ Department of Radiology, University of Wisconsin School of Medicine and Public Health, Madison, Wisconsin; ${ }^{2}$ University of \\ Wisconsin Carbone Cancer Center, Madison, Wisconsin; ${ }^{3}$ Department of Medicine, University of Wisconsin School of Medicine \\ and Public Health, Madison, Wisconsin; and ${ }^{4}$ Department of Medical Physics, University of Wisconsin, Madison, Wisconsin
}

\begin{abstract}
Estrogen receptor (ER) and progesterone receptor (PR) are important prognostic and predictive biomarkers in breast cancer. PET using ER- and PR-specific radioligands enables a wholebody, noninvasive assessment of receptor expression. Recent investigations of ER imaging with ${ }^{18} \mathrm{~F}$-fluoroestradiol have focused on diagnosing ER-positive metastatic disease, optimizing ER-targeted drug dosage, and predicting endocrine therapy benefit. Studies of PR imaging with ${ }^{18} \mathrm{~F}$-fluorofuranyl norprogesterone have investigated how imaging changes in PR expression as a downstream target of ER activation may reflect an early response to ER-targeted therapy. This focused review highlights recent achievements in preclinical and clinical imaging of ER and $\mathrm{PR}$ in breast cancer.
\end{abstract}

Key Words: estrogen receptor; progesterone receptor; PET/ CT; breast cancer

J Nucl Med 2020; 61:172-176

DOI: 10.2967/jnumed.119.228858

\section{$\mathbf{S}$} eroid hormone receptors function as ligand-activated nuclear transcription factors that regulate gene expression. Approximately $75 \%$ of breast cancers express estrogen receptor (ER) $\alpha$ and progesterone receptor (PR). Endocrine therapy that inhibits ER signaling is indicated for primary and metastatic ER-positive breast cancer. Immunohistochemistry remains the standard method for assessing ER and PR status. However, determination of receptor status from tissue biopsy can be prone to sampling error. Furthermore, not all metastatic sites are amenable to biopsy, and receptor expression across disease sites can vary within a patient. PET using ER- and PR-specific radioligands enables a whole-body, noninvasive assessment of receptor status. This information could potentially guide treatment decisions based on the presence and functionality of ER as a drug target. Imaging can also provide longitudinal information regarding ER and PR status, which can change over

Received Aug. 27, 2019; revision accepted Oct. 22, 2019.

For correspondence or reprints contact: Amy M. Fowler, University of Wisconsin, 600 Highland Ave., Madison, WI 53792.

E-mail: afowler@uwhealth.org

Published online Nov. 15, 2019.

COPYRIGHT (c) 2020 by the Society of Nuclear Medicine and Molecular Imaging. time after therapy. This focused review highlights publications within the last 5 y investigating preclinical and clinical imaging of ER and PR in breast cancer.

\section{${ }^{18}$ F-BASED RADIOLIGANDS FOR IMAGING ER}

The most studied radiopharmaceutical for imaging ER is $16 \alpha-{ }^{18}$ F-fluoro-17 $\beta$-estradiol, or ${ }^{18} \mathrm{~F}$-fluoroestradiol (Fig. 1). ${ }^{18} \mathrm{~F}$-fluoroestradiol is a radiolabeled steroid that binds to ER protein within target cells, similarly to endogenous estradiol. ${ }^{18}$ F-fluoroestradiol was developed at Washington University and the University of Illinois, with most clinical trials performed at Washington University and the University of Washington. These landmark studies investigated the correlation between in vitro ER assays and ${ }^{18} \mathrm{~F}$-fluoroestradiol uptake, heterogeneity of ER expression, and predictive value for endocrine therapy response. Additional work has been published from teams at Memorial Sloan Kettering Cancer Center, The Netherlands, China, and Canada. Details of these articles are summarized in multiple review articles (1-4). Recent investigations have built on this foundation and are highlighted herein.

\section{Assessment of ER Status}

Chae et al. recently published the largest prospective study comparing ${ }^{18}$ F-fluoroestradiol imaging with ER protein expression (5). They aimed to demonstrate agreement between qualitative ${ }^{18} \mathrm{~F}$-fluoroestradiol PET/CT interpretation and ER immunohistochemistry. The study population consisted of 85 patients with recurrent or metastatic breast cancer (47 ER-positive, 38 ER-negative). ER positivity was defined as an Allred score of at least 3 , which reflects the proportion of positive cells and staining intensity. ${ }^{18} \mathrm{~F}$-fluoroestradiol uptake was interpreted as positive if it was increased compared with background activity or negative if it was equivocal or decreased relative to background. Positive percentage agreement was $76.6 \% \quad(95 \%$ confidence interval [95\%CI], 62.0-87.7; $P=0.0018)$. Negative percentage agreement was $100 \%(95 \% \mathrm{CI}, 90.8-100 ; P=$ $0.00053)$. Interreader agreement was high at $0.90(95 \% \mathrm{CI}$, $0.78-1.0)$. The study concluded that ${ }^{18} \mathrm{~F}$-fluoroestradiol PET/ CT is safe and accurate for determining ER status.

In addition to visual assessment, quantitative ${ }^{18} \mathrm{~F}$-fluoroestradiol analyses for distinguishing ER-positive from ER-negative lesions 


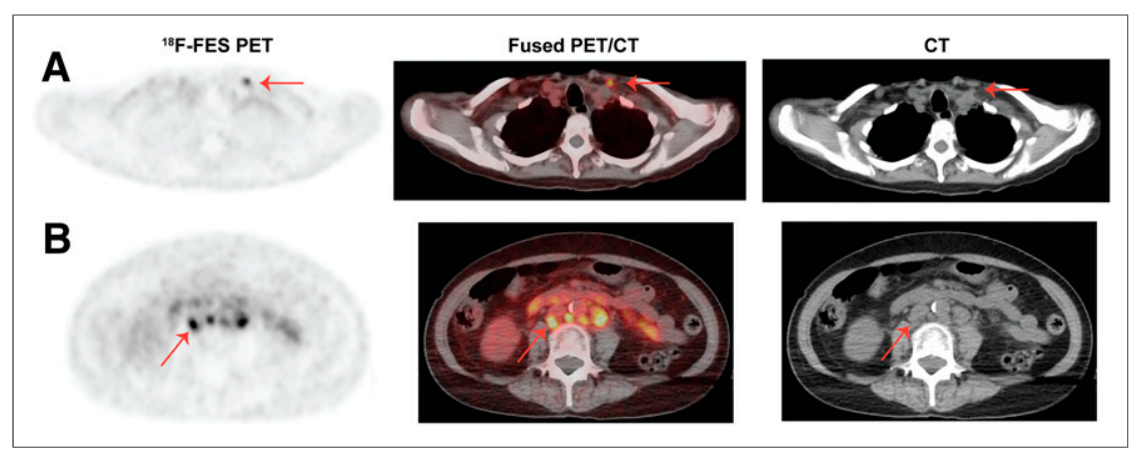

FIGURE 1. ${ }^{18} \mathrm{~F}$-fluoroestradiol PET/CT images of a postmenopausal woman with invasive ductal carcinoma (ER-positive, PR-positive, HER2-negative) and histologically confirmed metastatic disease show uptake in a left supraclavicular lymph node that does not meet size criteria for anatomic enlargement (A, arrow) and retroperitoneal lymph nodes $(B$, arrow). Physiologic clearance is noted in small bowel. that ${ }^{18} \mathrm{~F}$-fluoroestradiol specifically binds to ER, similarly to endogenous estradiol, has been provided through clinical studies correlating ${ }^{18} \mathrm{~F}$-fluoroestradiol uptake with ER protein expression, in vitro receptor ligand binding assays, and receptor blocking studies using coinjected nonradiolabeled estradiol. To confirm the binding specificity of ${ }^{18}$ F-fluoroestradiol, Salem et al. used-in engineered breast cancer cell lines - a mutagenesis approach that prevents ER from binding estradiol (10). Through substitution of glycine 521 for arginine in the ER ligand-binding pocket, ${ }^{18} \mathrm{~F}$-fluoroestradiol binding in cells and uptake in tumor xenografts was completely abolished.

have been performed. Using an $\mathrm{SUV}_{\max }$ of at least 1.5, the positive and negative percentage agreements were $85.1 \%$ and $78.9 \%$, respectively, as published by Chae et al. (5). In a metaanalysis of $5^{18} \mathrm{~F}$-fluoroestradiol imaging studies with ER immunohistochemistry results (143 patients), pooled sensitivity and specificity were $82 \%$ and $95 \%$, respectively (6). In another study of 13 heavily pretreated patients with metastatic ER-positive breast cancer, Venema et al. identified 1.54 as the optimal ${ }^{18} \mathrm{~F}$-fluoroestradiol $\mathrm{SUV}_{\max }$ cutoff to discriminate ER-positive from ER-negative lesions (7). In a retrospective study of 46 patients with histologically confirmed invasive breast cancer reported by Yang et al., the optimal ${ }^{18} \mathrm{~F}$-fluoroestradiol threshold was 1.21 for $\mathrm{SUV}_{\text {mean }}$ and 1.82 for $\mathrm{SUV}_{\max }(8)$. Thus, including previously published data, optimal ${ }^{18} \mathrm{~F}$-fluoroestradiol $\mathrm{SUV}_{\max }$ thresholds for distinguishing between ER-positive and ER-negative lesions ranged from 1.5 to $2.0(1-4,6)$.

Recent studies have also confirmed earlier evidence of a correlation between ${ }^{18} \mathrm{~F}$-fluoroestradiol uptake and ER protein expression. A strong association between ${ }^{18} \mathrm{~F}$-fluoroestradiol $\mathrm{SUV}_{\max }$ and the Allred score (correlation coefficient, 0.83; $P<0.0001$ ) was reported by Chae et al. (5). Similarly, Venema et al. reported a significant correlation between semiquantitative ER measurement using the H-score (0-300) and ${ }^{18}$ F-fluoroestradiol $\mathrm{SUV}_{\max }\left(R^{2}=0.78 ; P=0.01\right)(7)$. Furthermore, Yang et al. found a correlation coefficient of $0.726(P<0.001)$ between ER expression (scored $0,1+$, $2+$, or $3+$ ) and ${ }^{18}$ F-fluoroestradiol $\mathrm{SUV}_{\text {mean }}$ (using a $40 \%$ $\mathrm{SUV}_{\max }$ threshold), which was slightly higher than the correlation observed using $\mathrm{SUV}_{\text {max }}(r=0.691 ; P<0.001)$ (8). Gupta et al. reported a correlation coefficient of 0.767 (95\%CI, 0.266-0.942; $P=0.0096$ ) between ${ }^{18} \mathrm{~F}$-fluoroestradiol $\mathrm{SUV}_{\max }$ and ER (scored 15\%, 40\%, 90\%, and 100\%) (9). Overall, a strong correlation has been established between imaging and pathology despite different methods for ER protein quantification.

Binding specificity is necessary for establishing the analytic validity of ${ }^{18} \mathrm{~F}$-fluoroestradiol imaging. Evidence inferring
These results suggest that ${ }^{18} \mathrm{~F}$-fluoroestradiol retention in ERpositive breast cancer is strictly dependent on an intact ligandbinding pocket with high specificity.

Given the importance of the ER ligand binding domain for ${ }^{18} \mathrm{~F}$-fluoroestradiol binding, frequently identified hotspot mutations within this region of ER in patients with metastatic breast cancer raised questions regarding a potential effect on the diagnostic accuracy of ${ }^{18} \mathrm{~F}$-fluoroestradiol imaging. These mutations in the ER gene (ESRl) are biologically significant since they exhibit constitutively active ER transcriptional function with partial resistance to endocrine therapy and reduced binding affinity to estradiol, and they correlate with reduced patient survival (11). Kumar et al. created breast cancer cell lines expressing wild-type or mutant ER to test whether activating ESRl mutations impact the accuracy of ${ }^{18} \mathrm{~F}$-fluoroestradiol PET imaging for identifying ER-positive lesions (12). The study showed that ESR1 mutations occurring at tyrosine 537 are not detrimental to ${ }^{18} \mathrm{~F}$-fluoroestradiol binding or uptake in cells and tumor xenografts. These results support ${ }^{18} \mathrm{~F}$-fluoroestradiol imaging as a valid option for ER-positive breast cancer with these ESR1 mutations.

Additional phase II data regarding the diagnostic accuracy of ${ }^{18} \mathrm{~F}$-fluoroestradiol imaging have been published by Paquette et al. (13). The performance of a newer ER-targeted radiopharmaceutical, 4-fluoro-11 $\beta$-methoxy-16 $\alpha-{ }^{18}$ F-fluoroestradiol, or ${ }^{18} \mathrm{~F}$-4FMfluoroestradiol, was compared with ${ }^{18} \mathrm{~F}$-fluoroestradiol in 31 patients with ER-positive breast cancer. Decreased background uptake on PET/CT and improved tumor contrast were observed for ${ }^{18} \mathrm{~F}$-4FMfluoroestradiol compared with ${ }^{18}$ F-fluoroestradiol, which may improve diagnostic confidence and reduce false-negative results.

\section{Imaging ER Heterogeneity in Metastatic Disease}

Site-to-site ER heterogeneity across metastases is evident with ${ }^{18}$ F-fluoroestradiol imaging. Nienhuis et al. retrospectively analyzed ${ }^{18} \mathrm{~F}$-fluoroestradiol PET/CT scans for 91 patients (1,617 lesions) with metastatic ER-positive breast cancer (14). They found that $36 \%$ of patients had both 
${ }^{18} \mathrm{~F}$-fluoroestradiol-positive and ${ }^{18} \mathrm{~F}$-fluoroestradiol-negative lesions, which is within the previously published range of $28 \%-45 \%(1-4)$. Aside from the interesting biologic phenomena this finding may represent, such as clonal evolution and influence of the metastatic tumor microenvironment, it has potentially important clinical implications. Since targeting the site of biopsy to confirm metastatic disease in a patient with multiple lesions is based on lesion size and on safety or accessibility, different treatment approaches may be pursued with differing outcomes in patients with site-tosite heterogeneity in ER expression.

\section{Image-Guided Dosing of ER Antagonists}

${ }^{18} \mathrm{~F}$-fluoroestradiol imaging allows quantification of receptor binding during ER antagonist treatment. This approach can be used in dose escalation trials to maximize treatment benefits while minimizing side effects. Using a preclinical ER-positive breast cancer model, Heidari et al. demonstrated reduced tumor uptake of ${ }^{18} \mathrm{~F}$-fluoroestradiol correlating with decreased ER expression in a dose-dependent manner with fulvestrant, thus tracking early efficacy of ER blockade and degradation (15). This approach was investigated by van Kruchten et al. in a prospective study of 16 patients with metastatic ER-positive breast cancer (16). They tested whether the current standard dosing of fulvestrant optimally blocks tumor ER availability by performing ${ }^{18}$ F-fluoroestradiol PET/CT at baseline and during treatment. Incomplete blockade of ER was observed in $38 \%$ of patients and was associated with early disease progression. Thus, therapeutic monitoring via ${ }^{18} \mathrm{~F}$-fluoroestradiol imaging could reveal ineffective ER antagonist dosing and potentially allow for dose adjustment or switching to an alternative drug.

During novel drug development, serial ${ }^{18} \mathrm{~F}$-fluoroestradiol imaging can be useful for selecting the optimal dosage to achieve ER suppression while minimizing side effects. GDC-0810, a novel ER antagonist and degrader, was investigated in a phase I trial involving 30 patients with ERpositive metastatic breast cancer (17). The optimal dosage for phase II evaluation was determined by measuring the reduction in ${ }^{18} \mathrm{~F}$-fluoroestradiol uptake during treatment, reflecting ER occupancy and downregulation. As a substudy of a therapeutic trial of Z-endoxifen (an active metabolite of tamoxifen), ${ }^{18}$ F-fluoroestradiol PET/CT was performed at baseline and 1-5 d after treatment initiation in 8 patients with a variety of ER-positive solid tumors (18). The study found a $33.6 \%$ decrease $(P=0.0078)$ in the average $\mathrm{SUV}_{\max }$ as early as $1 \mathrm{~d}$ after Z-endoxifen administration. Thus, ${ }^{18} \mathrm{~F}$-fluoroestradiol imaging could serve as a useful pharmacodynamic marker for evaluating ER antagonist therapies.

\section{Predicting Endocrine Therapy Response}

Another clinically relevant application of ${ }^{18} \mathrm{~F}$-fluoroestradiol imaging is predicting response to endocrine therapy. In the neoadjuvant setting, 2 recent trials have incorporated baseline ${ }^{18} \mathrm{~F}$-fluoroestradiol PET/CT in the study design.
The Neo-ALL-IN trial tested the aromatase inhibitor, letrozole, in combination with a human epidermal growth factor receptor 2 (HER2)-targeted drug, lapatinib, in postmenopausal patients with stage II-III, ER-positive, HER2-positive breast cancer administered before surgery (19). Baseline ${ }^{18} \mathrm{~F}$ fluoroestradiol PET/CT was performed for 24 patients. An $\mathrm{SUV}_{\text {max }}$ of greater than 5.5 significantly correlated with clinical overall response rate $(P=0.007)$. For the NEOCENT trial, postmenopausal patients with ER-rich primary invasive breast cancer were randomized to either neoadjuvant chemotherapy (5-fluorouracil, epirubicin, and cyclophosphamide) or neoadjuvant endocrine therapy (letrozole) before surgery (20). Baseline ${ }^{18} \mathrm{~F}$-fluoroestradiol PET/CT was performed for 26 patients (13 randomized to chemotherapy, 13 randomized to endocrine therapy). Two patients in the chemotherapy cohort had visually negative ${ }^{18} \mathrm{~F}$-fluoroestradiol imaging, and both achieved a pathologic complete response. None of the 10 evaluable patients in the chemotherapy cohort with ${ }^{18}$ F-fluoroestradiol-avid tumors achieved a pathologic complete response. None of the 5 patients in the endocrine therapy cohort with an $\mathrm{SUV}_{\max }$ of less than 7.3 were pathologic responders, whereas 5 of the 7 patients in the chemotherapy group with an $\mathrm{SUV}_{\max }$ of less than 7.3 were pathologic responders $(P=0.03)$. These data imply that neoadjuvant chemotherapy could be superior to endocrine therapy in tumors with low ${ }^{18} \mathrm{~F}$-fluoroestradiol uptake, indicating nonfunctional ER. Together, these studies suggest that ${ }^{18}$ F-fluoroestradiol imaging could be helpful for neoadjuvant therapy selection.

In the metastatic setting, a study by van Kruchten et al. assessed the predictive value of ${ }^{18} \mathrm{~F}$-fluoroestradiol imaging for response to estradiol treatment, a therapeutic approach for extensively pretreated patients (21). Patients with acquired endocrine-resistant metastatic ER-positive breast cancer and progression after at least 2 lines of therapy underwent baseline ${ }^{18} \mathrm{~F}$-fluoroestradiol PET/CT followed by estradiol treatment. For 15 evaluable patients, the positive predictive value was $60 \%$, the negative predictive value was $80 \%$, and the area under the curve was 0.62 using a median $\mathrm{SUV}_{\max }$ cutoff of 1.5 for discriminating clinical benefit from progressive disease. The study highlighted the potential for ${ }^{18} \mathrm{~F}$-fluoroestradiol imaging to identify patients with acquired endocrine resistance who are unlikely to benefit from estradiol therapy indicated by poor ${ }^{18} \mathrm{~F}$-fluoroestradiol uptake. This study adds to landmark studies from Washington University and the University of Washington, which demonstrated a predictive value of ${ }^{18} \mathrm{~F}$-fluoroestradiol imaging for endocrine therapy benefit $(1-4)$.

Current multicenter trials aim to validate the predictive accuracy for endocrine therapy response in the metastatic setting. The ECOG/ACRIN EAI142 trial (NCT02398773) in the United States is designed to determine the negative predictive value of ${ }^{18} \mathrm{~F}$-fluoroestradiol PET/CT for clinical benefit at 6 mo in 99 patients with ER-positive metastatic breast cancer treated with first-line endocrine therapy. Ongoing European multicenter studies include 
the TRANSCAN ET-FES randomized trial (EUDRACT 2013000-287-29) and the IMPACT-MBC trial (NCT01957332) in The Netherlands.

\section{Prognostic Significance of ${ }^{18} \mathrm{~F}$-Fluoroestradiol Imaging}

Additional prognostic information may be gained from both ${ }^{18} \mathrm{~F}$-fluoroestradiol and ${ }^{18} \mathrm{~F}$-FDG imaging. Kurland et al. investigated ${ }^{18} \mathrm{~F}$-fluoroestradiol and ${ }^{18} \mathrm{~F}$-FDG as combined predictors of progression-free-survival in 84 evaluable patients with ER-positive breast cancer undergoing endocrine therapy for primary, recurrent, or metastatic disease (22). The study identified 3 groups with distinct outcomes. Metabolically indolent disease (low ${ }^{18}$ F-FDG uptake) corresponded with the longest median progression-free-survival (26.1 mo; 95\%CI, 11.2-49.7). Metabolically aggressive but strongly ER-positive disease $\left({ }^{18} \mathrm{~F}\right.$-FDG high; ${ }^{18} \mathrm{~F}$-fluoroestradiol high) corresponded with intermediate progression-free-survival (7.9 mo; 95\%CI, 5.6-11.8). Metabolically aggressive and weakly ER-positive disease $\left({ }^{18} \mathrm{~F}\right.$-FDG high; ${ }^{18} \mathrm{~F}$-fluoroestradiol low) corresponded with the shortest progression-freesurvival (3.3 mo; 95\%CI, 1.4-not evaluable). Thus, prognostic information provided by ${ }^{18} \mathrm{~F}$-fluoroestradiol imaging may be most impactful for patients with metabolically aggressive disease.

\section{Future of ER Imaging}

Robust evidence exists to support the clinical use of ${ }^{18} \mathrm{~F}$ fluoroestradiol imaging to determine ER status in metastatic breast cancer for endocrine therapy selection. ${ }^{18} \mathrm{~F}$-fluoroestradiol is currently clinically approved for this indication only in France. In 2019, Zionexa submitted a new-drug application for ${ }^{18} \mathrm{~F}$-fluoroestradiol (EstroTep) to the U.S. Food and Drug Administration. After approval, important next steps for clinical ${ }^{18}$ F-fluoroestradiol imaging include development of practice guidelines detailing patient preparation, scan parameters, expected physiologic distribution, tracer uptake quantification, scan interpretation, and standardized reporting (23).

Another critical step will be establishing payer coverage. In a simulation study, ${ }^{18}$ F-fluoroestradiol PET/CT appears

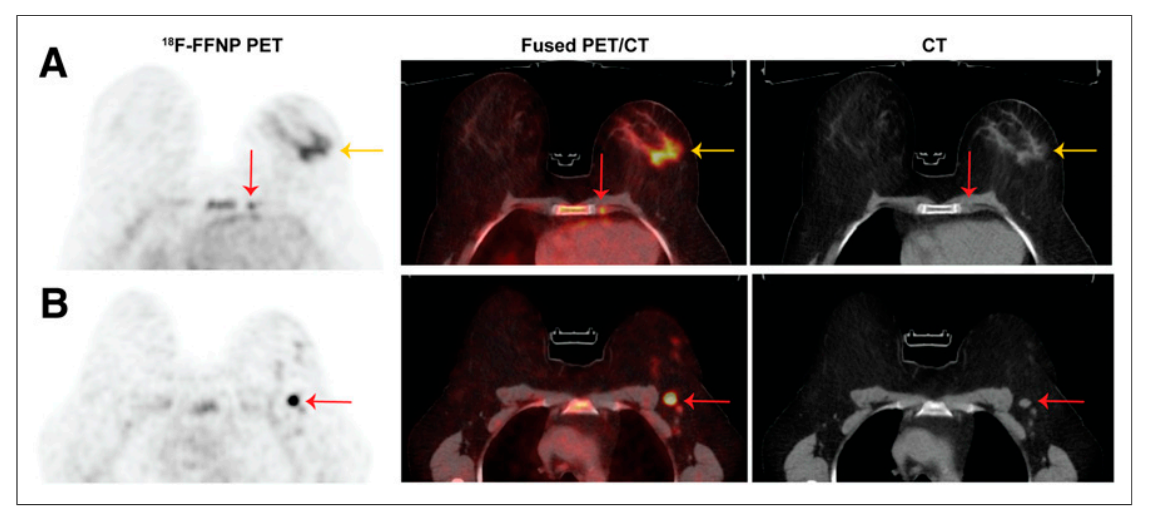

FIGURE 2. ${ }^{18} \mathrm{~F}-\mathrm{FFNP}$ PET/CT images of a postmenopausal woman with invasive lobular carcinoma (grade 2, ER-positive, PR-positive, HER2-negative) show uptake in biopsy-proven left breast malignancy (A, yellow arrow), left internal mammary lymph node ( $A$, red arrow) and left level 1 axillary lymph node (B, arrow). to be cost effective as an initial imaging test for diagnosing ER-positive metastatic breast cancer by decreasing the number of biopsies and false-negative results compared with conventional work-up (24). Additional cost-effectiveness studies are needed.

\section{IMAGING PR USING ${ }^{18}$ F-BASED RADIOLIGANDS}

$\mathrm{PR}$ is an estrogen-regulated target gene and can be an indicator of ER functionality. In this paradigm, activated ER induces PR gene transcription and ultimately increases PR protein. When antagonists inhibit ER function, PR messenger RNA and protein decrease.

The most studied PR-targeted radiopharmaceutical is ${ }^{18} \mathrm{~F}$-fluorofuranyl norprogesterone ( ${ }^{18} \mathrm{~F}$-FFNP) (Fig. 2). ${ }^{18} \mathrm{~F}$ FFNP is a radiolabeled progestin analog that binds to PR protein within target cells, similarly to endogenous progesterone. ${ }^{18}$ F-FFNP was developed at Washington University and the University of Illinois, and the first-in-humans study was performed at Washington University (25). This study demonstrated initial safety and feasibility and is the only clinical study using this tracer published to date.

Preclinical studies have quantified binding parameters of ${ }^{18}$ F-FFNP for PR in breast cancer cells. Using T47D cells, which endogenously express both $\mathrm{A}$ and $\mathrm{B}$ isoforms of PR, Salem et al. measured the equilibrium dissociation constant of ${ }^{18}$ F-FFNP to be $0.41 \pm 0.05 \mathrm{nM}$, with a half-maximal inhibitory concentration of $2.6 \mathrm{nM}(95 \% \mathrm{CI}, 2.0-3.4 \mathrm{nM})$, by competitive binding with the nonradioactive progestin, R5020 (26). Thus, ${ }^{18}$ F-FFNP exhibits strong binding affinity for PR. Using PR-negative MDA-MB-231 cells genetically engineered to express either PR-A or PR-B, there was no differential binding of ${ }^{18} \mathrm{~F}-\mathrm{FFNP}$ between the 2 isoforms (27). This is relevant since imbalanced isoform expression can influence prognosis and treatment outcomes (27).

Imaging changes in PR as a downstream target of ER activation may reflect an early response to endocrine therapy. Chan et al. showed that ${ }^{18} \mathrm{~F}-\mathrm{FFNP}$ imaging can measure changes in PR expression as a predictor of endocrine sensitivity (28). After estrogen deprivation via ovariectomy, endocrine-sensitive STAT1-deficient mouse mammary tumors exhibited an early decrease in ${ }^{18}$ F-FFNP uptake. However, endocrineresistant tumors had no significant change in ${ }^{18}$ F-FFNP uptake. Furthermore, ${ }^{18} \mathrm{~F}$-fluoroestradiol and ${ }^{18} \mathrm{~F}-\mathrm{FDG}$ uptake was unchanged, highlighting the unique information gained by serial ${ }^{18}$ F-FFNP imaging.

Additionally, Salem et al. used ${ }^{18} \mathrm{~F}$ FFNP to measure PR expression in endocrine-sensitive T47D cells after estradiol treatment, which activates ER (27). In both cells and tumor xenografts, ${ }^{18} \mathrm{~F}$-FFNP uptake increased after estradiol treatment. This preclinical 
study complements a recently completed clinical trial performed at Washington University (NCT02455453). The trial recruited 47 patients with ER-positive metastatic breast cancer who received baseline ${ }^{18} \mathrm{~F}$-FFNP PET/CT, treatment with low-dose estradiol for $24 \mathrm{~h}$, and then a repeat ${ }^{18} \mathrm{~F}$-FFNP PET/CT scan. The study aims to determine whether changes in ${ }^{18} \mathrm{~F}$-FFNP uptake after estradiol for $24 \mathrm{~h}$ predict a clinical benefit from standard endocrine therapy. The potential significance of this study is that ${ }^{18} \mathrm{~F}$-FFNP imaging may represent an early biomarker for endocrine therapy response.

\section{Future of PR Imaging}

Research continues into optimizing ${ }^{18} \mathrm{~F}$-FFNP production methods and developing novel agents for PR imaging. A new radiosynthesis technique has been reported to reduce the time required for ${ }^{18} \mathrm{~F}$-FFNP synthesis, as would be advantageous for high-volume facilities (29). Two new ${ }^{18} \mathrm{~F}$-labeled ethisterone derivatives, ${ }^{18} \mathrm{~F}$-EAEF and ${ }^{18} \mathrm{~F}$-FPTT, have been developed and tested in preclinical models (30,31). Additional preclinical and phase I studies will be needed to evaluate potential advantages of these agents over ${ }^{18}$ F-FFNP. As $\mathrm{PR}$ function in breast cancer continues to be revealed, ${ }^{18} \mathrm{~F}$ FFNP imaging may be useful for identifying PR-rich targets for combined ER and PR antagonists.

\section{DISCLOSURE}

Financial support was received from the Clinical and Translational Science Award program through the NIH National Center for Advancing Translational Sciences (grant 1UL1TR002373), the University of Wisconsin Institute of Clinical and Translational Research (KL2 scholar award 1KL2TR002374). The UW-Madison Department of Radiology receives in-kind research support from GE Healthcare. No other potential conflict of interest relevant to this article was reported.

\section{REFERENCES}

1. Allott L, Smith G, Aboagye EO, Carroll L. PET imaging of steroid hormone receptor expression. Mol Imaging. 2015;14:534-550.

2. Linden HM, Peterson LM, Fowler AM. Clinical potential of estrogen and progesterone receptor imaging. PET Clin. 2018;13:415-422.

3. Liao GJ, Clark AS, Schubert EK, Mankoff DA. ${ }^{18}$ F-fluoroestradiol PET: current status and potential future clinical applications. J Nucl Med. 2016;57:1269-1275.

4. Talbot JN, Gligorov J, Nataf V, et al. Current applications of PET imaging of sex hormone receptors with a fluorinated analogue of estradiol or of testosterone. $Q J$ Nucl Med Mol Imaging. 2015;59:4-17.

5. Chae SY, Ahn SH, Kim SB, et al. Diagnostic accuracy and safety of 16alpha$\left[{ }^{18} \mathrm{~F}\right]$ fluoro-17beta-oestradiol PET-CT for the assessment of oestrogen receptor status in recurrent or metastatic lesions in patients with breast cancer: a prospective cohort study. Lancet Oncol. 2019;20:546-555.

6. Evangelista L, Guarneri V, Conte PF. ${ }^{18} \mathrm{~F}$-fluoroestradiol positron emission tomography in breast cancer patients: systematic review of the literature \& metaanalysis. Curr Radiopharm. 2016;9:244-257.

7. Venema CM, Mammatas LH, Schroder CP, et al. Androgen and estrogen receptor imaging in metastatic breast cancer patients as a surrogate for tissue biopsies. J Nucl Med. 2017;58:1906-1912.

8. Yang Z, Sun Y, Xu X, et al. The assessment of estrogen receptor status and its intratumoral heterogeneity in patients with breast cancer by using ${ }^{18} \mathrm{~F}$-fluoroestradiol PET/CT. Clin Nucl Med. 2017;42:421-427.

9. Gupta M, Datta A, Choudhury PS, Dsouza M, Batra U, Mishra A. Can ${ }^{18}$ Ffluoroestradiol positron emission tomography become a new imaging standard in the estrogen receptor-positive breast cancer patient: a prospective comparative study with ${ }^{18} \mathrm{~F}$-fluorodeoxyglucose positron emission tomography? World J Nucl Med. 2017;16:133-139.

10. Salem K, Kumar M, Powers GL, et al. ${ }^{18} \mathrm{~F}-16$ alpha-17beta-fluoroestradiol binding specificity in estrogen receptor-positive breast cancer. Radiology. 2018;286:856-864.

11. Dustin D, Gu G, Fuqua SAW. ESR1 mutations in breast cancer. Cancer. 2019;125: 3714-3728.

12. Kumar M, Salem K, Michel C, Jeffery JJ, Yan Y, Fowler AM. ${ }^{18}$ F-fluoroestradiol PET imaging of activating estrogen receptor-alpha mutations in breast cancer. J Nucl Med. 2019;60:1247-1252.

13. Paquette M, Lavallee E, Phoenix S, et al. Improved estrogen receptor assessment by PET using the novel radiotracer ${ }^{18} \mathrm{~F}-4 \mathrm{FMFES}$ in estrogen receptorpositive breast cancer patients: an ongoing phase II clinical trial. J Nucl Med. 2018;59:197-203.

14. Nienhuis HH, van Kruchten M, Elias SG, et al. ${ }^{18}$ F-fluoroestradiol tumor uptake is heterogeneous and influenced by site of metastasis in breast cancer patients. J Nucl Med. 2018;59:1212-1218.

15. Heidari P, Deng F, Esfahani SA, et al. Pharmacodynamic imaging guides dosing of a selective estrogen receptor degrader. Clin Cancer Res. 2015;21:1340-1347.

16. van Kruchten M, de Vries EG, Glaudemans AW, et al. Measuring residual estrogen receptor availability during fulvestrant therapy in patients with metastatic breast cancer. Cancer Discov. 2015;5:72-81.

17. Wang Y, Ayres KL, Goldman DA, et al. ${ }^{18}$ F-fluoroestradiol PET/CT measurement of estrogen receptor suppression during a phase I trial of the novel estrogen receptor-targeted therapeutic GDC-0810: using an imaging biomarker to guide drug dosage in subsequent trials. Clin Cancer Res. 2017;23:3053-3060.

18. Lin FI, Gonzalez EM, Kummar S, et al. Utility of ${ }^{18} \mathrm{~F}$-fluoroestradiol $\left({ }^{18} \mathrm{~F}-\mathrm{FES}\right)$ $\mathrm{PET} / \mathrm{CT}$ imaging as a pharmacodynamic marker in patients with refractory estrogen receptor-positive solid tumors receiving Z-endoxifen therapy. Eur $J$ Nucl Med Mol Imaging. 2017;44:500-508.

19. Park JH, Kang MJ, Ahn JH, et al. Phase II trial of neoadjuvant letrozole and lapatinib in Asian postmenopausal women with estrogen receptor (ER) and human epidermal growth factor receptor 2 (HER2)-positive breast cancer [Neo-ALL-IN]: highlighting the TILs, ER expressional change after neoadjuvant treatment, and FES-PET as potential significant biomarkers. Cancer Chemother Pharmacol. 2016;78:685-695.

20. Chae SY, Kim SB, Ahn SH, et al. A randomized feasibility study of ${ }^{18} \mathrm{~F}$ fluoroestradiol PET to predict pathologic response to neoadjuvant therapy in estrogen receptor-rich postmenopausal breast cancer. J Nucl Med. 2017;58:563-568.

21. van Kruchten M, Glaudemans A, de Vries EFJ, Schroder CP, de Vries EGE, Hospers GAP. Positron emission tomography of tumour $\left[{ }^{18} \mathrm{~F}\right]$ fluoroestradiol uptake in patients with acquired hormone-resistant metastatic breast cancer prior to oestradiol therapy. Eur J Nucl Med Mol Imaging. 2015;42:1674-1681.

22. Kurland BF, Peterson LM, Lee JH, et al. Estrogen receptor binding $\left({ }^{18} \mathrm{~F}-\mathrm{FES}\right.$ PET) and glycolytic activity $\left({ }^{18} \mathrm{~F}-\mathrm{FDG}\right.$ PET) predict progression-free survival on endocrine therapy in patients with ER+ breast cancer. Clin Cancer Res. 2017;23: $407-415$.

23. Venema CM, Apollonio G, Hospers GA, et al. Recommendations and technical aspects of 16alpha- $\left[{ }^{18} \mathrm{~F}\right]$ fluoro-17beta-estradiol PET to image the estrogen receptor in vivo: the Groningen experience. Clin Nucl Med. 2016;41:844-851.

24. Koleva-Kolarova RG, Greuter MJ, van Kruchten M, et al. The value of PET/CT with FES or FDG tracers in metastatic breast cancer: a computer simulation study in ER-positive patients. Br J Cancer. 2015;112:1617-1625.

25. Dehdashti F, Laforest R, Gao F, et al. Assessment of progesterone receptors in breast carcinoma by PET with $21{ }^{18} \mathrm{~F}$-fluoro- $16 \alpha, 17 \alpha-\left[(R)-\left(1^{\prime}-\alpha\right.\right.$-furylmethylidene) dioxy]-19-norpregn-4-ene-3,20-dione. J Nucl Med. 2012;53:363-370.

26. Salem K, Kumar M, Kloepping KC, Michel CJ, Yan Y, Fowler AM. Determination of binding affinity of molecular imaging agents for steroid hormone receptors in breast cancer. Am J Nucl Med Mol Imaging. 2018;8:119-126.

27. Salem K, Kumar M, Yan Y, et al. Sensitivity and isoform specificity of ${ }^{18} \mathrm{~F}-$ fluorofuranylnorprogesterone for measuring progesterone receptor protein response to estradiol challenge in breast cancer. J Nucl Med. 2019;60:220-226.

28. Chan SR, Fowler AM, Allen JA, et al. Longitudinal noninvasive imaging of progesterone receptor as a predictive biomarker of tumor responsiveness to estrogen deprivation therapy. Clin Cancer Res. 2015;21:1063-1070.

29. Basuli F, Zhang X, Blackman B, et al. Fluorine-18 labeled fluorofuranylnorprogesterone ( $\left.\left[{ }^{18} \mathrm{~F}\right] \mathrm{FFNP}\right)$ and dihydrotestosterone $\left(\left[{ }^{18} \mathrm{~F}\right] \mathrm{FDHT}\right)$ prepared by "Fluorination on Sep-Pak" method. Molecules. 2019;24:E2389.

30. Wu X, You L, Zhang D, et al. Synthesis and preliminary evaluation of a ${ }^{18} \mathrm{~F}$ labeled ethisterone derivative $\left[{ }^{18} \mathrm{~F}\right] \mathrm{EAEF}$ for progesterone receptor targeting. Chem Biol Drug Des. 2017;89:559-565.

31. Gao F, Peng C, Zhuang R, et al. ${ }^{18} \mathrm{~F}$-labeled ethisterone derivative for progesterone receptor targeted PET imaging of breast cancer. Nucl Med Biol. 2019;72-73: 62-69. 